\title{
Global efforts on vaccines for COVID-19: Since, sooner or later, we all will catch the coronavirus
}

\author{
RAJu MukHERJEe (1) \\ Department of Biology, Indian Institute of Science Education and Research (IISER) Tirupati, Tirupati \\ 517 507, India \\ (Email,raju.mukherjee@iisertirupati.ac.in) \\ MS received 3 April 2020; accepted 23 April 2020; published online 7 May 2020
}

\begin{abstract}
COVID-19 is an emerging infectious disease that has turned into a pandemic. It spreads through droplet transmission of the new coronavirus SARS-CoV-2. It is an RNA virus displaying a spike protein as the major surface protein with significant sequence similarity to SARS-CoV which causes severe acute respiratory syndrome. The receptor binding domain of the spike protein interacts with the human angiotensin converting enzyme 2 and is considered as the antigenic determinant for stimulating an immune response. While multiple candidate vaccines are currently under different stages of development, there are no known therapeutic interventions at the moment. This review describes the key genetic features that are being considered for generating vaccine candidates by employing innovative technologies. It also highlights the global efforts being undertaken to deliver vaccines for COVID-19 through unprecedented international cooperation and future challenges post development.
\end{abstract}

Keywords. COVID-19; SARS-CoV-2; coronavirus; vaccines

\section{Introduction}

'Companies that have the skill to be able to do it are not going to just sit around and have a warm facility, ready to go for when you need it', said Anthony Fauci, the director of the National Institute of Allergy and Infectious Diseases at NIH, USA, and a member of the White House Coronavirus Task Force, at a panel discussion (Florko 2020). He has been aware of the mood of vaccine-manufacturing Big Pharma, which has not changed much since the 1980 s and is largely driven by a few very pertinent questions. Will the demand fade away with the end of the outbreak? Will the people choose, sometimes due to foolish reasons of vaccine hesitancy, not to use the product? What would be the timeline to reach breakeven and create wealth? With negligible prospects for profits, most drug makers have

This article is part of the Topical Collection: COVID-19: Disease Biology \& Intervention. been neglecting emerging infectious disease areas. However, due to the present spread of the COVID-19 pandemic, which has claimed, at the time of writing this article, 217,192 lives and infected 3,117,204 individuals so far, across all the continents except Antarctica, the need for vaccines have rekindled new interests and with a higher enthusiasm (Johns Hopkins University 2020). This can be understood from the rapidity in response to contain the outbreak and designing modalities for treatment. According to reports from Institute Pasteur, Shanghai, the first notice on treatments provided for pneumonia from hospitals in Wuhan, China, was received on 30th December 2019. Within 8 days, the coronavirus was identified, and by 10 th January 2020 , the very first genome of the virus was shared by the University of Sydney on behalf of the Chinese Academy of Sciences (Fontanet 2020; Huang et al. 2020). Consequent to that, a number of efforts are being made to socially contain the outbreak, screen available drugs for repurposing for COVID-19 and design specific vaccines against the new 
coronavirus. This review highlights the genomic features of coronavirus that should be considered for vaccine design, current innovations in vaccine technology, global efforts, and future challenges in vaccine developments for COVID- 19 .

\section{COVID-19 and coronavirus}

COVID-19 is a new infectious disease of the respiratory tract with chances of developing fulminant pneumonia similar to the severe acute respiratory syndrome (SARS) and is caused by the new coronavirus (SARSCoV-2), which spreads through contact with respiratory droplets from nose and mouth (Chan et al. 2020; WHO 2020a). Notably, $80 \%$ of people who are exposed or infected do not develop the active disease and show mild symptoms of seasonal flu and recover without major treatment. However, its estimated rate of incidence is much higher and is in the range of $20-60 \%$ in comparison with $8 \%$ for flu, and initial observations concludes that those developing severe disease require hospitalization for a period twice as much as that for acute flu (Lenski 2020). It originated in the Hubei province of China in late 2019, and spread rapidly around the world through human-to-human transmission along the international air-travel routes. The case fatality rate for COVID-19 at the time of writing this article stands at 10 times more than $0.1 \%$, as reported for the seasonal flu (Lenski 2020). SARS-CoV-2 is the third member of the pathogenic coronavirus after SARS-CoV and MERS-CoV (causes the Middle East respiratory syndrome) that shows severe pathology in humans among few others that only cause mild clinical symptoms like the flu (Cui et al. 2019). In the absence of a clinically proven effective treatment regime, current therapies for COVID-19 mainly receive supportive care supplemented by different combinations of antiretroviral drugs. A few FDA approved drugs and immune therapies are being repurposed and currently in clinical trials at several stages and available only on compassionate grounds. They have been proven efficacious in controlling SARS, influenza, HIV, and the Ebola virus replication by either targeting the viral RNA polymerase or the poly-protein protease or by boosting host immunity as adjunct therapy (Harrison 2020). Convalescent plasma from recovered individuals and monoclonal antibody therapies, which showed some success during the Ebola outbreak in Western Africa, are being developed (van Griensven et al. 2016). These antibodies will also form the basis for the serological diagnosis of asymptomatic spreaders, which are absent at the moment. However, here we will restrict our discussion to the multiple vaccines being developed against SARS-CoV-2.

\section{Origin of SARS-CoV-2 and genomic insights for vaccine development}

From the entire initial set of five genome sequences available, it was concluded that the new coronavirus is 99.9\% identical among themselves, suggesting its recent entry into humans (Zhou et al. 2020). All the 3365 viral genomes known today form a tight cluster based on phylogeny and show evidences of only 20 mutational events, much lower than the expected rate of $10^{-4}$ substitutions per site per year. This slow evolution is despite carrying RNA as the genome and much to the advantage for vaccine researchers (Dong et al. 2020). The closest match with $88-96 \%$ overall identity is that of the bat-derived beta-coronaviruses, indicating a zoonotic origin of the disease, similar to SARS and MERS but genetically distinct from the respective coronaviruses (Andersen et al. 2020). Interestingly, genomic and evolutionary evidence places the coronavirus found in Malayan pangolins (Pangolin-CoV) as the next best match with greater than $91 \%$ identity, thus hinting at the anteater as a possible intermediate host to the new coronavirus after spillover from bats, like the palm civets and the dromedary camels in case of SARS-CoV and MERS-CoV, respectively (Zhang et al. 2020). Further, the study posits Pangolins-CoV as the possible common ancestor for the human and bat versions based on the key amino acid residues present in its surface protein and crucial for interaction with the host receptor. Recombination events among the small sub-genomic regions are very common in coronaviruses due to the high level of coinfection that exists in their hosts. Therefore, tracing the evolutionary history of coronaviruses can be quite challenging and debatable.

Its positive-strand RNA genome consists of six major open reading frames and accessory genes coding for the typical non-structural proteins, including the RNA-dependent RNA polymerase, proteases, and the structural proteins, including the matrix $(\mathrm{M})$, envelope $(\mathrm{E})$, spike $(\mathrm{S})$ and nucleoprotein $(\mathrm{N})$. The surface spike (S) glycoprotein is responsible for binding to the human cell surface receptor, followed by membrane fusion and entry (figure 1) (de Wit et al. 2016). This spike protein is also pivotal for determining host specificity, estimating the capacity of disease 

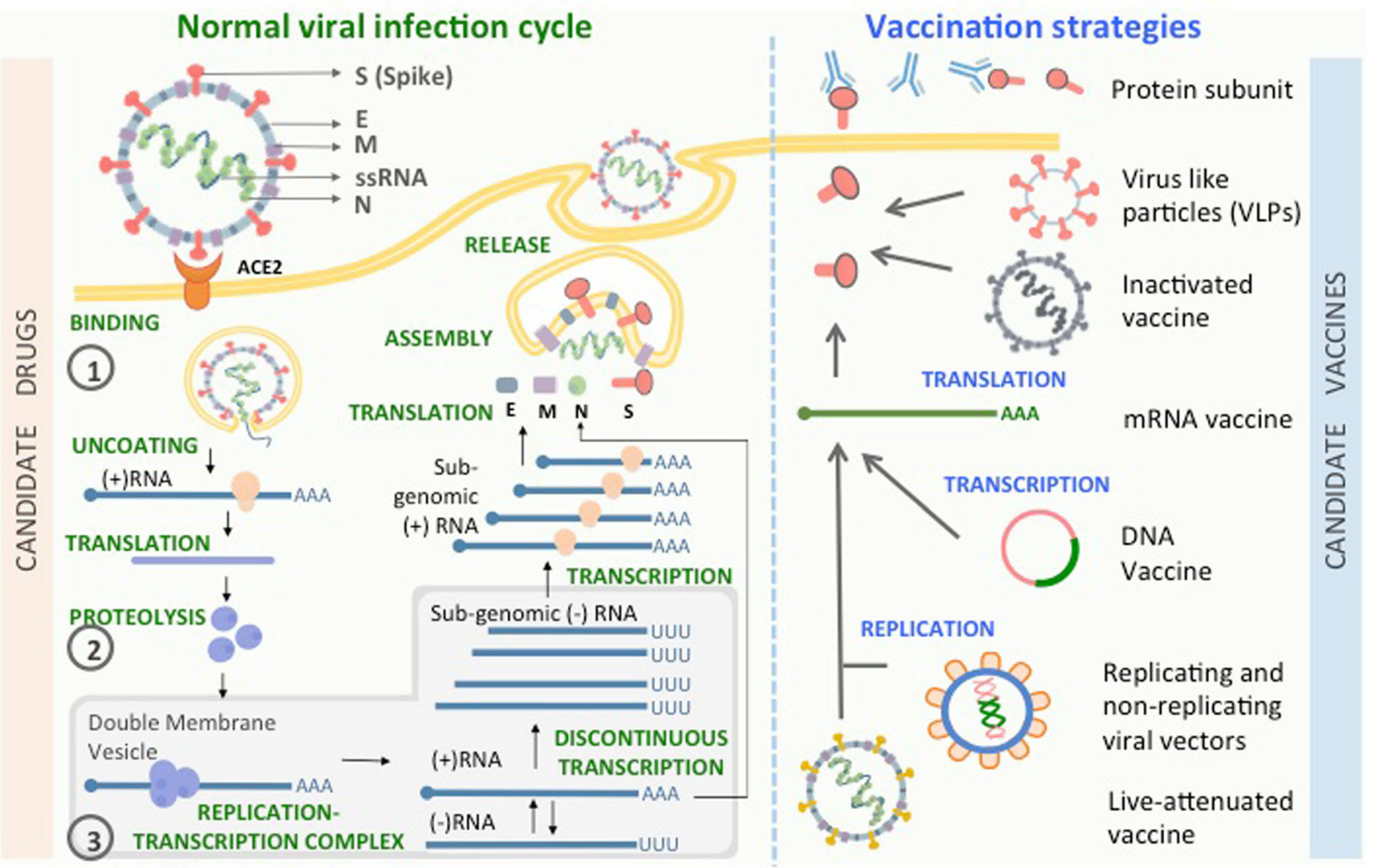

Figure 1. Potential vaccine candidates under development for COVID-19 treatment. The potential targets for small molecule drugs under clinical trails are annotated with numbers. 1, Chloroquine (endosomal acidification inhibitor); 2, Lopinavir-Ritonavir (protease inhibitor); 3, Remdesevir (reverse transcriptase inhibitor).

transmission, and for production of neutralizing antibody in human. Generally, the spike protein of coronaviruses consists of a signal peptide, extracellular, trans-membrane and intracellular regions. The hostrecognizing extracellular domain, responsible for cell membrane fusion, is divided by a polybasic cleavage site into a receptor binding $\mathrm{S} 1$ domain and the S2 domain (Andersen et al, 2020). When it comes to sequence identity at the amino acid level, which determines molecular recognition to the host receptor, the S1 domain shows only $68 \%$ identity in comparison with the bat version. Expectedly, phylogenetic analysis also places the receptor binding domain to a lineage closer to that of the SARS-CoV version, thus suggesting a binding to the same angiotensin-converting enzyme 2 (ACE2) (Lu et al. 2020; Zhou et al. 2020). Further, it underscores the emergence and reservoirs of SARS-CoV-2, which originated from bats but may have jumped into pangolins, with a plausible long period of natural selection in human or human-like ACE-2. The receptor binding domain appears to have accumulated mutation at five amino acid positions for optimizing binding to host following an early zoonotic transfer much before November 2019 (Andersen et al. 2020). An educated speculation indicates that a COVID-19-type epidemic may recur at any time in the future, by a different SARS-CoV-2 variant evolving from the early isolates under selective pressure in animals, further emphasizing the need for a vaccine.

\section{Identifying potential targets for designing vaccine candidates}

Vaccination strategies can be effectively used to reduce disease severity, control transmission by viral shedding and prevent future infections. Vaccines are usually directed to induce both arms of the adaptive immune response producing neutralizing antibody that prevents attachment to host cells and activate the body's $T$ killer cells that recognize and kill infected cells. Vaccine candidates are chosen from the various approaches available as per the level of protection required. The simplest method involves introducing a virus into the body after inactivation by chemicals, gamma irradiation or heat. Proteins expressed on the dead viral 
surface serves as antigen and induce the production of neutralizing antibodies as in the case of the early polio vaccine (figure 1) (Bandyopadhyay et al. 2015). Another method involves introducing weakened or attenuated versions of the virus that slowly replicates inside the host cell but provides enough viral antigen to induce an immune response, like the oral polio vaccine, rabies vaccine or the $\mathrm{BCG}$ vaccine given at birth to prevent childhood tuberculosis. Administering a nonvirulent organism is less safe than the inactivated virus and has been the cause for disease outbreak, but is much superior in terms of establishing a mild infection necessary for mounting a successful adaptive immune response (Afrough et al. 2019). Developing an attenuated vaccine in a short time is, however, challenging as it requires isolating a non-virulent strain through several rounds of time-consuming sub-culturing in the laboratory and requires adequate knowledge about the virulence factors expressed by the coronavirus. Furthermore, the level and type of adaptive immune response sometimes depend on the strain used in the product. One each of the above types is currently under preclinical development to estimate efficacy and toxicity: Formaldehyde-inactivated formulation is being developed by Sinovac Biotech in China, while a genetically modified poorly replicating coronavirus is being developed in a partnership between Codagenix, USA, and Serum Institute of India, India, using their proprietary deoptimization technology platform earlier used for developing vaccines for Zika, dengue and influenza (WHO 2020b).

A more targeted approach involves inducing adaptive immunity by introducing immunogenic viral proteins or their fragments to generate both antibodies and cell-mediated immune response. Delivering the antigenic epitopes with optimal levels of antigen presentation required for a broad protective immunity is vital for a successful immune response. The high degree of similarities in the genome, structural proteins, and even the host attachment receptor indicates the existence of a possible similarity in the immune responses against the two SARS coronaviruses. This also means that conclusions made from studies on SARS-CoV proteins might help in determining the immunogenic determinants required for vaccine development. In addition to the high level of potent antibodies against the $\mathrm{S}$ and $\mathrm{N}$ proteins of SARS-CoV, which were incidentally short-lived, strong T-cell responses that provide long term protections were detected for the same structural proteins (Tang et al. 2011). Intriguingly, when the experimentally identified dominant B-cell and T-cell epitopes of SARS-
CoV were mapped to the amino acid sequence of the spike and nucleocapsid proteins, 27 T-cell epitopes matched the SARS-CoV-2 proteins without a single mutation. Further, the epitopes located within the predicted receptor binding domain of the spike protein were associated with the most prevalent major histocompatibility complex (MHC) alleles in the human population (Ahmed et al. 2020). Similarly, a large number of B-cell epitopes found complete sequence identity with that of the nucleocapsid protein and both $\mathrm{S} 1$ and S2 domains of the spike protein. The prevalence of epitopes corresponding to the less exposed S2 domain, however, indicates its possible access to antibodies post attachment to the host cell. This analysis also helps preclude the apprehensions about the mismatches observed in the receptor binding region of the S1 domain from the structural analysis of the spike protein (Wrapp et al. 2020). Antibodies raised against the S2 domain of SARS-CoV spike protein may cross-react and neutralize SARS-CoV-2, thus positing an excellent opportunity for repurposing the candidate monoclonal antibodies that did not cross phase I trials for SARS vaccine development (Tian et al. 2020). Therefore, the spike protein of SARS$\mathrm{CoV}$ can be confidently placed as the most potent antigenic determinant against which candidate vaccines should be developed.

Another important consideration in designing vaccines, particularly in case of RNA viruses that generally have higher mutation rates, is the antigenic drift or genetic variability, which plays a pivotal role in choosing the best genome sequence of the viral strain for vaccine development, and this means we need to sequence the SARS-CoV-2 genome from as many regions possible from around the world (Dong et al. 2020). Choosing the right vaccine candidate is as much an art as a science since finding the viral components that will not trigger severe inflammation but can provide a protective immune memory is not trivial, more so when nothing much is known about the immune pathology of the disease and the immune evasion strategies employed by the virus to evade the host defense and most of our assumption is based on studies conducted on SARS-CoV.

\section{Innovations in vaccine technologies}

Several new vaccine generation methods, based on the concept of 'plug-and-play', have been developed that can be used to test candidates against multiple infections in a short time. This is possible primarily because 
the safety features are determined predominantly by the upstream and downstream process technology employed and not on the pathogen of interest. Vignettes of certain approaches using recombinant virus technology, nucleic acid vaccines, self-disseminating and protein vaccine approaches will be briefly described here (figure 1).

\subsection{Molecular clamps}

Since a virus is an obligate intracellular replicative entity that requires the host cell for its proliferation, all vaccine strategies are aimed at preventing the attachment of the virus to the host cell. At the molecular interface, this recognition is characterized by binding of the viral surface protein (Spike glycoprotein in SARS-CoV-2) to the receptor on human cells (ACE2) (Lu et al.). Antibodies generated against the surface protein antigen helps to neutralize this receptor binding, thus making protein subunit vaccines very popular. However, by themselves, these proteins would normally be unstable and may lose their molecular structure soon after injection, rendering it difficult for the immune cells to produce antibodies specific to the virus. Furthermore, the free-floating viral protein may change its shape to a post-recognition stable conformation, which is very different from the prerecognition state as displayed on the surface of the virus. The molecular clamping approach uses joining key structural polypeptides at several regions in the protein structure to efficiently retain the original shape of the immunogenic epitopes, thus stimulating a stronger response (UniQuest 2014).

\subsection{Replicating viral vectors}

With advances made in understanding the replication and pathogenesis of a wide variety of viruses along with the development of tools for genetic manipulation, it is now possible to generate safer attenuated vaccines expressing recombinant viral protein antigens from a proxy infection model (Robert-Guroff 2007). Replicating vectors in use includes adenovirus, measles virus, poxvirus and vesicular stomatitis virus (VSV) (Humphreys and Sebastian 2018). These viruses are either non-pathogenic in humans (VSV) or carry a genetically modified genome which neutralizes their virulent nature. The coding sequence for the required protein/subunit antigen is attached to the genome for optimal expression, assembly and packaging into the recombinant virus. Two major advantages underscore their potential as a vaccine of choice. Its ability to elicit a complete (innate and adaptive) immune response that is also tunable in terms of type and intensity of the response is its biggest advantage. The proxy natural infection model employed also allows the induction of co-stimulatory immune molecules that provide an adjuvant effect. Second, its replicative nature allows achieving similar protection at a 3-4 log lower dosage. Vectors based on measles virus have been considered for vaccines against HIV-AIDS and SARS due to its potential to provide lifelong immunity as a result of its ability to infect the antigen-presenting cells (dendritic cells and macrophages) (Zuniga et al. 2007).

\subsection{Non-replicating viral vectors}

In this case, the genes essential for replication of the adenovirus are deleted, among others, from the adenoviral genome and replaced with antigenic protein coding genes at the locus (Robert-Guroff 2007). The most popular Modified Vaccinia virus Ankara (MVA) is a natural attenuated version of poxvirus. Through repeated sub-culture in chicken cells, MVA has lost its ability to replicate and evade immune surveillance in human cells (Kennedy and Greenberg 2009). Replication-deficient vectors can accommodate larger gene inserts and also provide stability in terms of not regaining virulence when compared with the replication-competent versions.

\section{$5.4 \quad$ Virus-like particles (VLPS)}

One of the problems of using the protein subunit vaccine has been presenting the antigen in its most stable and effective conformation. VLPs are composed of multiple structural proteins, which upon recombinant expression have the ability to selfassemble into nanostructures enclosing the capsid proteins within itself (Syomin and Ilyin 2019). The VLPs can have a lipid envelope originating from the cell membrane producing them in the form of budding and can also be chimeric in nature, displaying an envelope protein from another virus. A wide range of production platforms expressing and correctly folding the antigenic proteins are available in bacterial and yeast systems, insect and mammalian cell lines and even in transgenic plants. In addition, since VLPs lack a genome of its own, they offer a similar level of stability as non-replicating vectors, thus offering the 
safety of subunit vaccine and efficacy of the live-attenuated vaccine, together.

\subsection{DNA vaccines}

Nucleic acid-based vaccines have emerged as a better alternative to live and inactivated vaccines for treatments against emerging pathogens as the DNA expressing the pathogen's protein can be produced in a short time. This does not involve growing the virus in the laboratory and gives equivalent protective immunity as other forms. In this case, the DNA sequence is introduced, often facilitated by nano-carriers, directly to the cells of a specific tissue. Once they make their way into the nucleus with the help of a targeting sequence, viral surface protein production, driven from an optimized promoter, is outsourced to the host cells natural machinery (Hobernik and Bros 2018). DNA vaccines are safe, stable and large quantities can be produced in a short time through cost-effective manufacturing processes, which is crucial for distribution in low-income regions.

\section{6 $m R N A$ vaccines}

First identified while studying immunization with tumor antigens for cancer therapy, mRNA vaccines are now extensively studied for emerging pathogens. Like DNA vaccines, they represent the new generation therapies and, being mRNA in nature, avoid the risk of being integrated into the host genome, as a bonus. They come in two flavors, traditional mRNA-based vaccines and self-amplifying mRNA vaccines. Both work on the strategy of using the host cell transcription machinery to produce the target proteins and induce adaptive immunity (Pardi et al. 2018). While the former is replication-deficient and contains the antigen's mRNA, the latter codes for a modified genome of an RNA virus where the target antigen of choice replaces the viruses' native genes coding for the structural proteins. The resulting virus can express the heterologous antigenic genes in the cytoplasm in high numbers but cannot package itself into a functional virus, while the mRNA can amplify itself using the RNA dependent RNA polymerase coded in its genome (Geall et al. 2012).

All of the above innovations are being rapidly utilized to develop multiple vaccines against the new coronavirus. Several epidemiological models predict that the present pandemic will stay for quite some time, albeit in several small pockets around the world and reappear in the subsequent waves of infection. But, will coronavirus usher in a new era in developing innovative vaccines for emerging infectious disease beyond COVID-19? We will have to wait for the results of the clinical trials to come out.

\section{Multiple vaccines towards one single goal}

Vaccine development for infectious diseases is equally challenging, similar to drug development, and normally takes the same amount of time. In the case of COVID19 , this is even harder because of unknown disease pathogenesis, unavailability of a validated animal model, and success is dependent on the human clinical trials. Despite all this, the scale and severity of the current pandemic have attracted around 35 companies - Big Pharma, small biotechnology firms and university spinoffs - to develop a vaccine against SARSCoV-2 (Lee 2020; WHO 2020b). Thanks to the attractive funding from two government agencies of the USA, Biomedical Advanced Research and Development Authority (Department of Health, USA) and the National Institute of Allergy and Infectious Diseases (National Institutes of Health, USA), work has been initiated on as many as 44 candidate vaccines (table 1), which are currently in clinical and preclinical stages. The COVID-19 outbreak has also highlighted innovations in funding mechanism through the involvement of Coalition for Epidemic Preparedness Innovations (CEPI), a global alliance-based vaccine accelerator for emerging infectious diseases, founded in 2017 by the governments of India and Norway with initial support from the World Economic Forum and philanthropic organizations like the Bill and Melinda Gates Foundation, USA, and Wellcome Trust, UK. This generous funding has enabled several small biotechnology firms and academic institutions to triage their diverse candidates, which is essential in situations where it is hard to predict which one and at which stage will reach a cul-de-sac. The new candidates are not only based on traditional approaches, they are also being built on the existing vaccines and leveraging on platforms used to develop the SARS or the MERS vaccines. It is important to mention that although there are great similarities between the immunogenic epitopes of the two SARS-CoVs, vaccines for COVID-19 do not have to be similar to the ones proposed against other coronaviruses. The newly developed vaccine against the Ebola Zaire virus by GlaxoSmithKline, UK, was produced using the adenoviral vectors used for an HIV 
Table 1. Candidate vaccines against SARS-CoV-2 and their stages of development (adapted from WHO draft on vaccine candidates as on March 2020 (WHO 2020b))

\begin{tabular}{|c|c|c|}
\hline Type of Technology & $\begin{array}{c}\text { Type of candidate } \\
\text { (Clinical Trials Registry) }\end{array}$ & Company/Consortium/Institutes \\
\hline \multicolumn{3}{|c|}{ Phase-I clinical trials in humans (Toxicity and immunogenicity) } \\
\hline \multirow[t]{2}{*}{ Non-Replicating Viral Vector } & $\begin{array}{l}\text { Adenovirus Type } 5 \text { Vector } \\
\text { (ChiCTR2000030906) }\end{array}$ & $\begin{array}{l}\text { CanSino Biological Inc. and Academy of } \\
\text { Military Medical Sciences' Institute of } \\
\text { Biotechnology, Beijing }\end{array}$ \\
\hline & $\begin{array}{l}\text { ChAdOx1 } \\
\text { (NCT04324606) }\end{array}$ & University of Oxford \\
\hline RNA Vaccine & $\begin{array}{l}\text { Lipid nanoparticle encapsulated mRNA } \\
\text { (NCT04283461) }\end{array}$ & Moderna/NIAID \\
\hline \multicolumn{3}{|c|}{ Preclinical development and toxicity } \\
\hline Inactivated Vaccine & Formaldehyde-inactivated with alum & Sinovac \\
\hline Live attenuated Vaccine & De-optimized live attenuated vaccine & Codagenix/Serum Institute of India \\
\hline Non-Replicating Viral Vector & MVA encoded VLP & GeoVax/BravoVax \\
\hline \multirow[t]{3}{*}{ Vaccine } & $\begin{array}{l}\text { Ad26 (alone or with MVA boost) } \\
\text { adenovirus-based NasoVAX expressing } \\
\text { SARS2-CoV spike protein }\end{array}$ & $\begin{array}{l}\text { Janssen Pharmaceutical } \\
\text { Altimmune }\end{array}$ \\
\hline & Ad5 $\mathrm{S}$ & Greffex \\
\hline & Oral Vaccine platform & Vaxart \\
\hline \multirow[t]{4}{*}{ Replicating Viral Vector } & Measles Vector & Zydus Cadila \\
\hline & & Institute Pasteur/Themis/Univ. of Pittsburg \\
\hline & Horsepox vector expressing S protein & Tonix Pharma/Southern Research \\
\hline & $\begin{array}{l}\text { Drosophila S2 insect cell expression } \\
\text { system VLPs }\end{array}$ & ExpreS2ion \\
\hline \multirow{13}{*}{$\begin{array}{l}\text { Protein Subunit } \\
\text { Vaccine }\end{array}$} & $\mathrm{S}$ protein & AJ Vaccines \\
\hline & & $\begin{array}{l}\text { WRAIR/USAMRIID EpiVax/Univ. of } \\
\text { Georgia }\end{array}$ \\
\hline & Adjuvanted S protein trimer & $\begin{array}{l}\text { Clover Biopharmaceuticals/Glaxo Smith } \\
\text { Kline }\end{array}$ \\
\hline & Peptide & Vaxil Bio \\
\hline & Ii-Key peptide & Generex/EpiVax \\
\hline & $\begin{array}{l}\text { Adjuvanted S protein (Baculovirus } \\
\text { production) }\end{array}$ & Sanofi Pasteur/Glaxo Smith Kline \\
\hline & $\begin{array}{l}\text { Full length S-trimer/nanoparticle }+ \\
\text { Matrix M }\end{array}$ & NovaVax \\
\hline & gp-96 backbone & Heat Biologics/Univ. Of Miami \\
\hline & Molecular clamp stabilized Spike protein & University of Queensland/Glaxo Smith Kline \\
\hline & $\mathrm{S} 1$ or $\mathrm{RBD}$ protein & Baylor College of Medicine \\
\hline & Subunit protein, plant produced & iBio/CC-Pharming \\
\hline & Subunit & VIDO-InterVac, University of Saskatchewan \\
\hline & Adjuvanted microsphere peptide & University of Saskatchewan \\
\hline \multirow[t]{2}{*}{ DNA Vaccine } & $\begin{array}{l}\text { DNA plasmid vaccine Electroporation } \\
\text { device }\end{array}$ & Inovio Pharmaceuticals \\
\hline & DNA plasmid vaccine & $\begin{array}{l}\text { Takis/Applied DNA Sciences/Evvivax } \\
\text { Zydus Cadila, India }\end{array}$ \\
\hline \multirow[t]{6}{*}{ RNA Vaccine } & $\begin{array}{l}\text { LNP-encapsulated mRNA encoding RBD } \\
\text { and } \\
\text { LNP-encapsulated mRNA cocktail } \\
\text { encoding VLP }\end{array}$ & $\begin{array}{l}\text { Fudan University/Shanghai JiaoTong } \\
\text { University/RNACure Biopharma }\end{array}$ \\
\hline & mRNA & China CDC/Tongji University/Stermina \\
\hline & mRNA & Arcturus/Duke-NUS \\
\hline & mRNA & BioNTech/Fosun Pharma/Pfizer \\
\hline & Self amplifying RNA & Imperial College London \\
\hline & mRNA & Curevac \\
\hline Virus like particle & Plant-derived VLP & Medicago \\
\hline
\end{tabular}


vaccine (GSK 2016). Institute Pasteur has proposed to use the measles vaccine virus vector backbone used earlier to prepare vaccines for Ebola, Zika and Lassa fever (WHO 2020b).

Phase I trials for toxicity and immunogenicity have started on healthy adult volunteers, with funding from CEPI, for a lipid nanoparticle encapsulated mRNA vaccine (mRNA-1273) co-developed by NIH's Virus Research Centre, NIAID and Moderna Inc. Another frontrunner in starting the clinical trials is CanSino Biologics in partnership with China's Academy of Military Medical Sciences' Institute of Biotechnology. This candidate is based on a non-replicating adenoviral vector used for Ebola, while University of Oxford's Jenner Institute has proposed to start phase I trial for a non-replicating adenoviral vector-based vaccine backbone (ChAdOx1) due to its proven efficacy among older people with underlying co-morbidities (Devlin 2020). Among others, candidates that are undergoing preclinical testing are mRNA-based vaccines from Germany's CureVac (funded by European Union and CEPI) and BioNTech (co-developed with Pfizer). Both companies are repurposing their leads from personalized cancer treatments to deliver vaccines that are tunable as per the level of immune response required (Curevac 2020; Pfizer 2020). Like Moderna, another American company Novavax has redeveloped a spike protein-based vaccine for SARS in combination with its proprietary matrix adjuvants. Institute Pasteur is leading a consortium with University of Pittsburgh and Themis Biosciences, Austria, to work on a replicating measles vaccine virus vector technology, based on SARS-CoV (Pasteur Institute 2020). With the availability of a high-resolution molecular structure of the trimeric spike protein, researchers at the University of Queensland, Australia, are using their disruptive molecular clamp technology, which helps the viral proteins to maintain their shape, thus enabling the immune system to mount a stronger response (University of Queensland 2020). It is being combined with GlaxoSmithKline's proprietary adjuvants in order to generate a long-lasting immune response. A lower adjuvanted dose allows immunization of a larger number of individuals with a low amount of the protein product. The same adjuvant-based potentiation is also being offered for Clover Biopharmaceutical's trimeric spike protein vaccine candidate. A very promising and widely discussed DNA vaccine candidate (INO-4800), being developed by Inovio Pharmaceuticals, has succeeded in raising funds from CEPI. Although DNAbased vaccines have not received any regulatory approvals in the past, and the company has not delivered any vaccine since its inception, it has committed to start clinical trials in April 2020 and has received support from the Bill and Melinda Gates Foundation to develop an intradermal electroporation device for vaccine delivery (Linnane 2020).

Intriguingly, large-scale phase III randomized control trials have started in Australia and Netherlands to check for the effectiveness of the BCG vaccine, a live-attenuated strain of Mycobacterium that causes tuberculosis in cattle, in reducing incidences and disease severity among the healthcare workers exposed to SARS-CoV2 (Byrne 2020). This recent development stems from a report which claimed to have found an excellent correlation on country-wise severity of COVID-19 symptoms to its national immunization program for protection against childhood $\mathrm{TB}$ and $\mathrm{TB}$ meningitis (Miller et al. 2020). It is also not surprising to note that countries practicing compulsory BCG vaccination are also socio-economically backward, therefore reports of low morbidity and mortality from COVID-19 may be related to low co-morbidities contributed by lifestyle diseases and not necessarily a protection offered by BCG vaccination. The study draws a link between high mortalities in Iran, which started BCG vaccination later in 1984, with the lack of non-specific protection among the older population. The non-specific protection by BCG against viral infections is plausibly offered by the induction of innate immune memory, again a very new concept in itself, that results in enhanced production of immune modulators and cytotoxic activity of immune cells. Nevertheless, if true, this might help to provide some levels of protection until a new specific vaccine is made available (Moorlag et al. 2019). However, it would be interesting to learn about the outcome of these large trials with respect to the degree of protection achieved as different strains of BCG is known to provide a wide range of efficacies in the case of tuberculosis in adults.

\section{How soon will we have a new vaccine?}

Usually, it takes 10-15 years of development through the classical path using inactivated or live attenuated vaccines after generating long-term efficacy data. During the Ebola emergency, this time was brought down to 5 years for candidates that are based on viral vector vaccines and involved running a few key steps of phase II trials in parallel. As the COVID-19 contagion rapidly engulfs a quarter of the world's population, Inovio Pharmaceutical has ambitiously accelerated this timeline to publish the human clinical 
trials data by the Fall of 2020, which is remarkably less than a year (Inovio Pharmaceuticals 2020). This is also expected, keeping in mind that there might not be enough patient volunteers available to participate in the human trials later. However, the test cohort should not miss out on including different age groups, ethnicity, gender and pregnant women. Finally, data needs to be collected for any rare occurrence of immunization-mediated enhancement of severe illness after infection. This is primarily caused by the development of sub-neutralizing antibodies, which promotes excessive viral entry to the host cells (Wan et al. 2020). However, when it comes to manufacturing and delivery, the process faces myriad of new challenges as often experienced by the pharmaceutical industry. Large quantities of the candidate vaccine are generally required to start a full-scale phase III trial and immunizations thereafter; moreover, each product will require a customized scale-up technology that will have to be developed. More and more pharmaceutical companies have shifted focus to the risk-averse lifestyle disease, and this has resulted in their losing the skillset and expertise required for vaccine development. This needs to be re-built by continuous financial commitments with no immediate guarantee for rewards and an unprecedented level of international cooperation, which is thankfully quite visible. Another important question that may arise and needs some clarity is selecting the target cohort for immunization. Healthier individuals might be naturally protected and more vulnerable while the needy older population might show problems of a poor immune response and require higher doses. Certainly, healthcare workers need them the most in our preparedness for the next wave of infection and perhaps also the traders in the wet markets if they continue to be in business.

In conclusion, once we have the vaccine and hopefully the apex of the pandemic is over, an effective policy needs to be carefully prepared to develop a global access framework for equitable distribution in the developing world while protecting intellectual property and market rights. Distribution of the vaccines should be made as far as possible based on humanitarian benevolence, centrally through international agencies including Global Alliance for Vaccines and Immunization (GAVI), UNICEF and WHO. Allowing the vaccine producing countries or the richest economies to stockpile the doses for the native population in the absence of a national emergency, as seen in the case of H1N1 influenza virus vaccine in 2009, will jeopardize all the momentum gained thus far (Fidler 2010).

\section{Acknowledgements}

The author would like to thank Dibya Saha for help in preparing illustrations amid country-wide lockdown and SERB, India, for research funding.

\section{Funding}

Funding was provided by SERB (Grant No. ECR/ 2016/000665).

\section{References}

Afrough B, Dowall S and Hewson R 2019 Emerging viruses and current strategies for vaccine intervention. Clin. Exp. Immunol. 196 157-166

Ahmed SF, Quadeer AA and McKay MR 2020 Preliminary identification of potential vaccine targets for the COVID19 coronavirus (SARS-CoV-2) based on SARS-CoV immunological studies. Viruses 12 https://doi.org/10. 3390/v12030254

Andersen KG, Rambaut A, Lipkin WI, Holmes EC and Garry RF 2020 The proximal origin of SARS-CoV-2. Nat. Med. https://doi.org/10.1038/s41591-020-0820-9

Bandyopadhyay AS, Garon J, Seib K and Orenstein WA 2015 Polio vaccination: past, present and future. Future Microbiol. 10 791-808

Byrne B 2020 Murdoch Children's Research Institute to trial preventative vaccine for COVID-19 healthcare workers, Murdoch Children's Research Institute

Chan JF, Yuan S, Kok KH, To KK, Chu H, et al. 2020 A familial cluster of pneumonia associated with the 2019 novel coronavirus indicating person-to-person transmission: a study of a family cluster. Lancet 395 514-523

Cui J, Li F and Shi ZL 2019 Origin and evolution of pathogenic coronaviruses. Nat. Rev. Microbiol. 17 181-192

Curevac 2020 CureVac focuses on the development of mRNA-based coronavirus vaccine to protect people worldwide, Curevac

de Wit E, van Doremalen N, Falzarano D and Munster VJ 2016 SARS and MERS: recent insights into emerging coronaviruses. Nat. Rev. Microbiol. 14 523-534

Devlin H 2020 UK scientists enrol volunteers for coronavirus vaccine trial. The Guardian March 27

Dong E, Du H and Gardner L 2020 An interactive web-based dashboard to track COVID-19 in real time. Lancet Infect. Dis. http://doi.org/10.1016/S1473-3099(20)30120-1 
Fidler DP 2010 Negotiating equitable access to influenza vaccines: global health diplomacy and the controversies surrounding avian influenza $\mathrm{H} 5 \mathrm{~N} 1$ and pandemic influenza H1N1. PLoS Med. 7 e1000247

Florko N 2020 Major drug makers haven't stepped up to manufacture NIH coronavirus vaccine, top U.S. health official says. STATnews February 11

Fontanet A 2020 COVID-19: What we have learnt as of 20 February 2020 by Arnaud Fontanet, Institute Pasteur

Geall AJ, Verma A, Otten GR, Shaw CA, Hekele A, et al. 2012 Nonviral delivery of self-amplifying RNA vaccines. Proc. Natl. Acad. Sci USA 109 14604-14609

GSK 2016 Update on GSK's response to the Zika virus disease outbreak., gsk.com

Harrison C 2020 Coronavirus puts drug repurposing on the fast track. Nat. Biotechnol. http://doi.org/10.1038/ d41587-020-00003-1

Hobernik D and Bros M 2018 DNA vaccines-how far from clinical use? Int. J. Mol. Sci. 19 10.3390/ijms 19113605

Huang C, Wang Y, Li X, Ren L, Zhao J, et al. 2020 Clinical features of patients infected with 2019 novel coronavirus in Wuhan, China. Lancet 395 497-506

Humphreys IR and Sebastian S 2018 Novel viral vectors in infectious diseases. Immunology 153 1-9

Johns Hopkins University 2020 (https://coronavirus.jhu.edu.)

Jiang SHY and Liu S 2005 SARS vaccine development. Emerg. Infect. Dis. 11. http://www.cdc.gov/eid

Kennedy JS and Greenberg RN 2009 IMVAMUNE: modified vaccinia Ankara strain as an attenuated smallpox vaccine. Expert Rev. Vaccines 8 13-24

Lee J 2020 These 16 companies are working on coronavirus treatments or vaccines - here's where things stand. MarketWatch March 24

Lenski RE 2020 Expert analyses of the SARS-CoV-2 coronavirus outbreak. Telliamedrevisited.wordpress.com February 22

Linnane C 2020 Inovio gets \$5 million grant from Gates Foundation to fund testing of device for delivering COVID-19 vaccine. MarketWatch March 12

$\mathrm{Lu}$ R, Zhao X, Li J, Niu P, Yang B et al. 2020 Genomic characterisation and epidemiology of 2019 novel coronavirus: implications for virus origins and receptor binding. Lancet 395 565-574

Miller A, Reandelar MJ, Fasciglione K, Roumenova V, Li Y and Otazu GH 2020 Correlation between universal BCG vaccination policy and reduced morbidity and mortality for COVID-19: an epidemiological study. medRxiv https://doi.org/10.1101/2020.03.24.20042937

Moorlag S, Arts RJW, van Crevel R and Netea MG 2019 Non-specific effects of BCG vaccine on viral infections. Clin. Microbiol. Infect. 25 1473-1478
Pardi N, Hogan MJ, Porter FW and Weissman D 2018 mRNA vaccines - a new era in vaccinology. Nat. Rev. Drug Discov. 17 261-279

Pasteur Institute 2020 CEPI collaborates with the Institut Pasteur in a consortium to develop COVID-19 vaccine pasteur.fr March 19

Pfizer Inc 2020 Pfizer and BioNTech to co-develop potential COVID-19 vaccine

Inovio Pharmaceuticals 2020 Inovio accelerates timeline for COVID-19 DNA vaccine INO-4800. Press release, ir.inovio.com March 3

Robert-Guroff M 2007 Replicating and non-replicating viral vectors for vaccine development. Curr. Opin. Biotechnol. 18 546-556

Syomin BV and Ilyin YV 2019 Virus-like particles as an instrument of vaccine production. Mol. Biol. 53 323-334

Tang F, Quan Y, Xin ZT, Wrammert J, Ma MJ, et al. 2011 Lack of peripheral memory B cell responses in recovered patients with severe acute respiratory syndrome: a sixyear follow-up study. J. Immunol. 186 7264-7268

Tian X, Li C, Huang A, Xia S, Lu S. et al. 2020 Potent binding of 2019 novel coronavirus spike protein by a SARS coronavirus-specific human monoclonal antibody. Emerg. Microbes Infect. 9 382-385

UniQuest 2014 Molecular clamp: a novel protein vaccine for influenza, RSV, Ebola and other human and veterinary viruses (http://www.pharmalicensing.com)

University of Queensland 2020 Significant step' in COVID19 vaccine quest. uq.edu.au February 21

van Griensven J, Edwards T, de Lamballerie X, Semple MG, Gallian P, et al. 2016 Evaluation of convalescent plasma for ebola virus disease in Guinea. N. Engl. J. Med. 374 33-42

Wan Y, Shang J, Sun S, Tai W, Chen J, et al. 2020 Molecular mechanism for antibody-dependent enhancement of coronavirus entry. $J$. Virol. 94

WHO 2020a Coronavirus disease (COVID-19) pandemic. who.int March 27

WHO 2020b DRAFT landscape of COVID-19 candidate vaccines. who.int March 20

Wrapp D, Wang N, Corbett KS, Goldsmith JA, Hsieh CL, et al. 2020 Cryo-EM structure of the 2019-nCoV spike in the prefusion conformation. Science 367 1260-1263

Zhang T, Wu Q and Zhang Z 2020 Probable Pangolin Origin of SARS-CoV-2 associated with the COVID-19 outbreak. Curr. Biol. 30 1346-1351 e1342

Zhou P, Yang XL, Wang XG, Hu B, Zhang L et al. 2020 A pneumonia outbreak associated with a new coronavirus of probable bat origin. Nature 579 270-273

Zuniga A, Wang Z, Liniger M, Hangartner L, Caballero M, et al. 2007 Attenuated measles virus as a vaccine vector. Vaccine 25 2974-2983 